\title{
On the development of integrated sensor for multiphase measurements
}

\author{
W. H. Ahmed, A. Al-Sarkhi, M. El-Nakla \& H. M. Badr \\ Department of Mechanical Engineering, \\ King Fahd University of Petroleum and Minerals, \\ Dhahran, Saudi Arabia
}

\begin{abstract}
The need for multiphase flow measurement in the oil and gas production and petrochemical industries has been significantly increased over the last few years. Reliable measurements of the multiphase flow parameters such as void fraction, phase concentration, phase velocity and flow pattern identification are important for accurate modelling and/or in the operation of multiphase systems. Although many multiphase flow meters were recently developed, challenges in measuring multiphase flow components remain unresolved. Therefore, extensive research efforts were spent in designing accurate multiphase flow meters and several meters are currently under development worldwide. However, due to the complexity of the multiphase flow mixture and in some cases when three or more phases co-exist, it is impossible to adopt only one technique to develop a multiphase flow meter. Consequently, the integration of multiple sensors, based on several measurement techniques, found to be the optimum solution for accurate multiphase flow metering. The main objective of this study is to evaluate existing multiphase flow measurement techniques for the potential integration in a multiphase flow metering device.

Keywords: multiphase flow, capacitance sensors, resistivity probes, integrated sensor, particle image velocimetery (PIV).
\end{abstract}

\section{Introduction}

The problem of metering oil-water-gas mixtures has been of great interest to the petroleum industry since the 1980s and considerable research and development works have been conducted to develop a three-phase flow meter suitable for use 
at the industrial level [1]. It is preferred that such a meter needs to be reasonably accurate, non-intrusive, reliable, flow regime independent, and appropriate for use over the whole range of volume fraction of all phases. Despite the large number of metering devices, methods and solutions that have been proposed previously, obtaining all of the above requirements remains a challenging task.

Reliable measurements of multiphase flow parameters such as void fraction, phase concentration, phase velocity and flow pattern identification are important for accurate modelling of multiphase systems. These parameters can be measured using a number of techniques, including radiation attenuation ( $\mathrm{X}$ or gamma-ray or neutron beams) for line or area averaged values, optical or electrical contact probes for local void fraction, impedance technique using capacitance sensors and direct volume measurement using quick-closing valves. The use of the different techniques depends on the applications, and whether a volumetric average or a local void fraction measurement is desired.

Recently, several oil production industries showed a great interest for active participation of researchers towards the development of multi-phase flow meters and better integration and selection of appropriate sensing elements. Although, the selection of a multiphase flow meter is the main challenge, the criteria for best selection of a multiphase flow meter should be in mind before starting the selection process. Some of these are the number of sensors in use, the complexity of the design, the maintenance cost, the difficulty of calibration process and finally the control and data processing. In addition, numerous of technical problems are typically encountered in the operation of such meters. For example, meters equipped with gamma densitometers utilizing a nuclear source to measure the fluid density involve environmental and safety issues associated with the nuclear sources. The coriolis meters, used to measure the mixture density by tracking the natural frequency of the vibrating pipe carrying the fluid, require a vibration source that makes them mechanically complex and relatively difficult to maintain. Also, the optical sensors such as fiber optics have demonstrated high accuracy in addition of being neither intrusive nor invasive. However, they can't deal with build-up particles caused by crude oil. Moreover, electrical sensors such as capacitance and conductance-based sensors may offer a faster time response than the optical sensors, however they lack the high accuracy and the good signal-to-noise ratio.

The available multiphase flow meters for multi-components gas-liquid-liquid flow are found in three different categories. These categories include separation meters, in-line meters and advanced signal processing systems combined with techniques for parameter estimation. The separation type multiphase meters are characterized by performing a complete or partial separation of the multiphase stream, followed by in-line measurement of each of the phases. On the other hand, the in-line multiphase meters are characterized by direct measurements of phase fractions and the phase flow rates are performed directly without any separation process. The meters commonly employ a combination of two or more of the measurement techniques.

Several studies reported the development of multiphase flow meters using different measuring techniques [2-6]. Although, there are a large number of 
multiphase meters developed in the past, the need for more accurate and suitable instrumentations for on-line monitoring and measurement of multiphase flows continues to increase. This is mainly due the challenging behaviour of multiphase flows that are characterized by turbulence, deformable phase interface, phase interaction, phase slip and compressibility of the gas phase. In addition, multiphase multi-component such as a three or four-component flow meter is considered to be of high importance to three main industries: oil, petrochemical and water treatment plants. It should be noted that a significant number of studies on multiphase flow measurements have been developed over the years and it will be difficult to cover the literature on all techniques in this section. Therefore, only a review related to the specific techniques used in the present study will be presented.

\subsection{Particle Image Velocimetry (PIV)}

The Particle Image Velocimetry (PIV) is a non-intrusive laser optical measurement technique for research and diagnostics of flows, turbulence, microfluidics, spray atomization and combustion processes. PIV has the ability to measure whole field velocity instantaneously. This technique flourishes in recent decades based on the improvement of its hardware components and image processing. Improved image processing algorithms enhances the measurement precision, reliability, and spatial resolution [7]. The PIV has been widely applied to investigate flow velocity fields in two- and three-phase flows. Tokuhiro et al. [8] examined the flow field around an oscillating bubble and its boundary of a turbulent flow around the oscillating bubble with the application of PIV. Brücker [9] studied the wake generated by a single and a couple of rising ellipsoidal bubbles. Oritz-Villafuerte et al. [9] and Hassan et al. [10] focused their research on the turbulent motion generated in a continuous liquid phase with threedimensional PIV.

In applying PIV to multiphase flow systems, one might encounter special problems that do not exist in single phase flow. One problem arises at high values of the concentration of the dispersed phase. This may cause a strong attenuation of the laser light, so that the penetration length of the light sheet into the flow system is limited. Another problem originates from the difference in the velocity distributions of the continuous and dispersed phases [11]. If we evaluate the PIV recording in a conventional way, e.g. with a correlation technique, then the resulting velocity value will be affected by the signals from the two (or more) different phases (i.e. some average of two (or more) different velocities). Merzkirch et al. [12] have investigated experimentally possible ways for solving these problems. Moreover, they have provided different solutions of separating the signals arising from the individual phases, so that phase-separated velocity distributions can be determined.

Recently, Zhou et al. [13] have used the PIV technique successfully to measure the velocity fields of gas-droplet-solid multiphase flow in the experimental setup of a novel compact semidry flue gas desulfurization process with a multifluid alkaline spray generator. They presented detailed experimental data that provided an improved understanding of flow behaviour, mixing 
characteristic, and interface interaction of multiphase flow both in the confined alkaline spray generator and in the duct bent pipe section.

\subsection{Capacitance measurements technique}

The principle of the capacitance method is based on the differences in the dielectric constants of the two phases in the flow, and the capacitance measured across the sensors is dependent on the volume ratio of the two phases. A review of the applications and different designs of capacitance sensors for two-phase flow measurements are reported by Ahmed and Ismail [6]. They identified several disadvantages of the impedance technique, which are sometimes difficult to resolve. For example, the capacitance measurement is sensitive to the void fraction distribution or flow regimes due to the non-uniformity of the electrical field inside the measuring volume. These disadvantages can be compensated by identifying the multiphase flow pattern. The capacitance measurement is also sensitive to the changes in electrical properties of the two phases due to temperature. Also, the noise due to the electromagnetic field around the sensor and connecting wires can significantly affect the signal and needs to be minimized through proper design of the sensor shield. Detailed study on capacitance sensor design optimization for best performance was presented earlier by Ahmed [14].

The sensitivity of the capacitance meter was also improved by Andrade [15]. He used a full power supply potential swing on the shield electrodes to allow for the use of simpler shield electrode design. Using the full swing improves the capacitance background and balances such noises as the wire capacitances. Furthermore, it is difficult to resolve the change in phase distribution within the sensor measurement volume. The sensitivity to the flow pattern can be increased through better design of the sensors. For example, Hung et al. [16] used eight electrodes along the circumference of the tube to obtain a tomographic image of the two-phases. On the other hand, homogenizing the electric field in the axial direction minimizes the error due to the void distribution in the measuring volume. This could be done by using rotating type sensors as suggested by Merilo et al. [17]. The electrical capacitance tomography technique was later implemented by Gamio et al. [18] to image various two-phase gas-oil horizontal flows in a pressurized pipeline. They emphasized the potential of this technique for real-time flow visualization and flow regime identification in practical industrial application at high pressure operating conditions. On the other hand, Jiang and Xiong [19] introduced a novel non-invasive electrostatic method for measurement of the solid velocity and mass flow rate in gas-solid flow. In their system, measurements of flow velocity and mass flow rate were realized by an electrostatic sensor capable of detecting the electrostatic signals of solid powder in the gas-solid flow. The transit time of powder was measured by crosscorrelating electrostatic signals, and the velocity was calculated. By correlating the voltage on electrostatic sensor and the fixed mass flow rate, the voltage-mass flow rate curve of the sensing system was established. Jiang and Xiong [19] concluded that electrostatic method can be used to trace and measure the velocity 
of the solid phase, and the method is capable of performing in-line measurements of the mass flow rate of the gas-solid flow.

\section{Integrated sensor for air-water-oil measurements}

The present idea is related to a metering system that is used to identify the flow pattern, phase fraction and velocity of multiphase flow using capacitanceresistance measuring techniques. This device consists of two holders (A) and (B) separated by distance (Z) as shown in Figure 1. Each holder carries a multiple of capacitance and resistance probes distributed over the pipe circumferential as shown in Figure 2. The distance between each probe holder can be adjusted

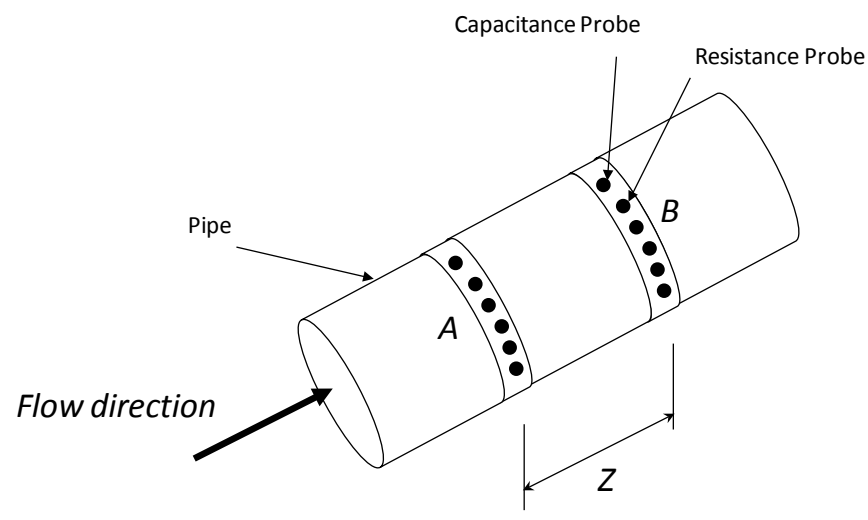

Figure 1: $\quad$ Device main components.

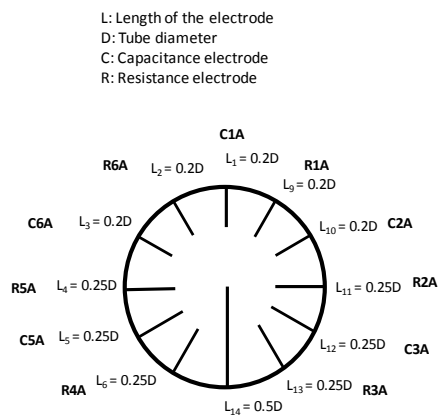

C4A

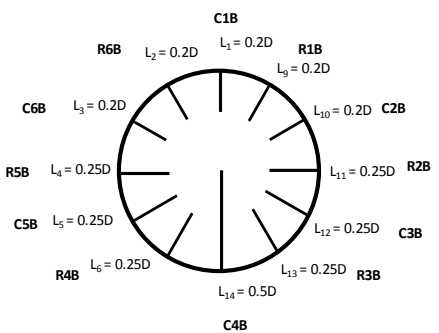

Station (B)

Station (A)

Flow direction inside to the page and angle between electrodes is $30 \mathrm{deg}$

Figure 2: Distribution of capacitance and resistance probes along the pipe circumferential. 
based on the pipe diameter (D) and flow velocity ranges. The length of each intrusive probe is varied along the pipe circumferential selected to detect any flow pattern expected in the pipe. The probe signal will be function of the fraction of each phase in contact and between the electrodes (Figure 3). The reason for using two different types of probes at the same cross section is to detect the existence of three-phase flow with reasonable accuracy. This is mainly because the difference in the capacitance response (calculated from Equations (1) through (4)) between the water and gas or water and oil flows can be distinguished unlike for oil and gas flows as shown in Figure 4. On the other hand, the resistance probes provide better signal for oil-gas flows as calculated from Equations (5) through (8) and shown in Figure 5. Simulation of multiphase flow signal consists of air-water-oil passing through both capacitance and resistance probes as shown in Figure 6. It can be seen that the oil and air phases

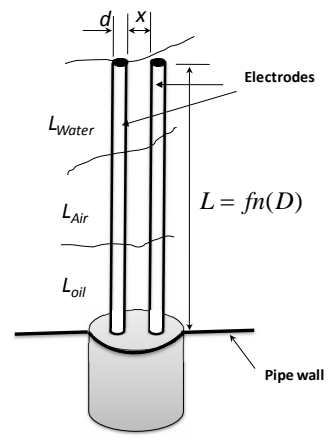

Figure 3: $\quad$ Probe dimensions.

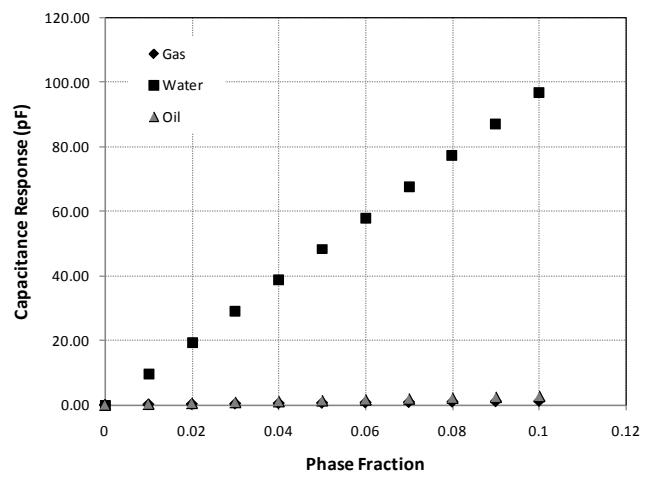

Figure 4: $\quad$ Capacitance probe response at different phase fraction. 


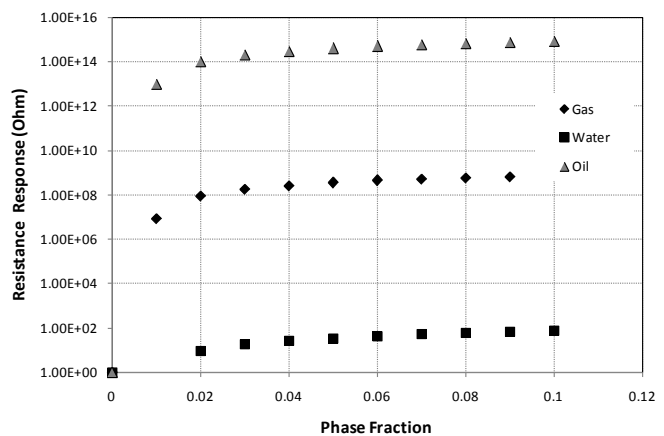

Figure 5: $\quad$ Resistance probe response at different phase fraction.

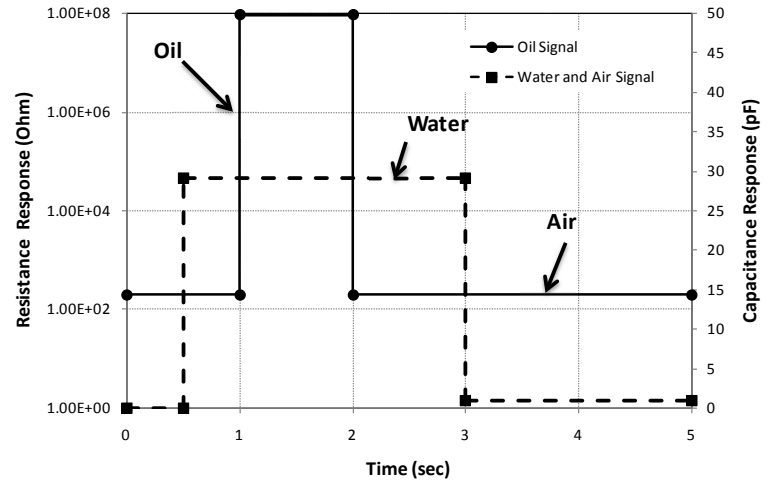

Figure 6: Difference in the response of both resistance and capacitance probes for multiphase flow signal.

can be clearly identified by the resistance probe while the water is recognized by the capacitance probes.

$$
\begin{gathered}
C_{1 A-a i r}=\frac{\pi L_{\text {air }} \cdot k \varepsilon_{O}}{\ln \left[(x / 2 d)+\sqrt{(x / 2 d)^{2}-1}\right]} \\
C_{1 A-\text { water }}=\frac{\pi L_{\text {water }} \cdot k \varepsilon_{O}}{\ln \left[(x / 2 d)+\sqrt{(x / 2 d)^{2}-1}\right]} \\
C_{1 A-\text { oil }}=\frac{\pi L_{\text {oil }} \cdot k \varepsilon_{o}}{\ln \left[(x / 2 d)+\sqrt{(x / 2 d)^{2}-1}\right]} \\
C_{1 A-\text { total }}=C_{1 A-\text { water }}+C_{1 A-\text { air }}+C_{1 A-\text { oil }}
\end{gathered}
$$

The resistance between two parallel cylinders can be expressed as:

$$
R_{1 A-\text { air }}=\frac{L_{\text {air }} R_{O}}{\pi} \cosh ^{-1}(x / d)
$$




$$
\begin{gathered}
R_{1 A-\text { water }}=\frac{L_{\text {water }} R_{O}}{\pi} \cosh ^{-1}(x / d) \\
R_{1 A-\text { oil }}=\frac{L_{\text {oil }} R_{O}}{\pi} \cosh ^{-1}(x / d) \\
R_{\text {total }}=\frac{1}{R_{1 A-\text { air }}}+\frac{1}{R_{1 A-\text { water }}}+\frac{1}{R_{1 A-\text { oil }}}
\end{gathered}
$$

This device can be installed in any flow direction (vertical, horizontal or inclined) and is used to identify different flow patterns including stratified, stratified wavy, annular, slug and bubble flow. The signal obtained from each probe can be used to determine the liquid holdup or gas void fraction in addition to liquid level. The combined signals of all capacitance or resistance probes can be used to determine the phase distribution around the pipe. Also, the instantaneous signals obtained at both stations (A) and (B) can be correlated to determine the frequency of slugging, slug length and translational velocity.

Signals for capacitance probes (C1A to C6A) are collected as shown in Figure 7 using data acquisition station (A) and analyzed individually to determine the value of the water and gas fractions at the corresponding circumferential location. At the same time, the signals from the resistance probes (R1A to R6A) (also Figure 7) are collected to determine the content of the oil phase in the multiphase mixture. Average phase fraction can also be obtained from both capacitance and resistance probes. Additional signal processing is performed between each electrode pairs and cross correlation technique is applied to determine more information of the phase distribution and velocity of phases.

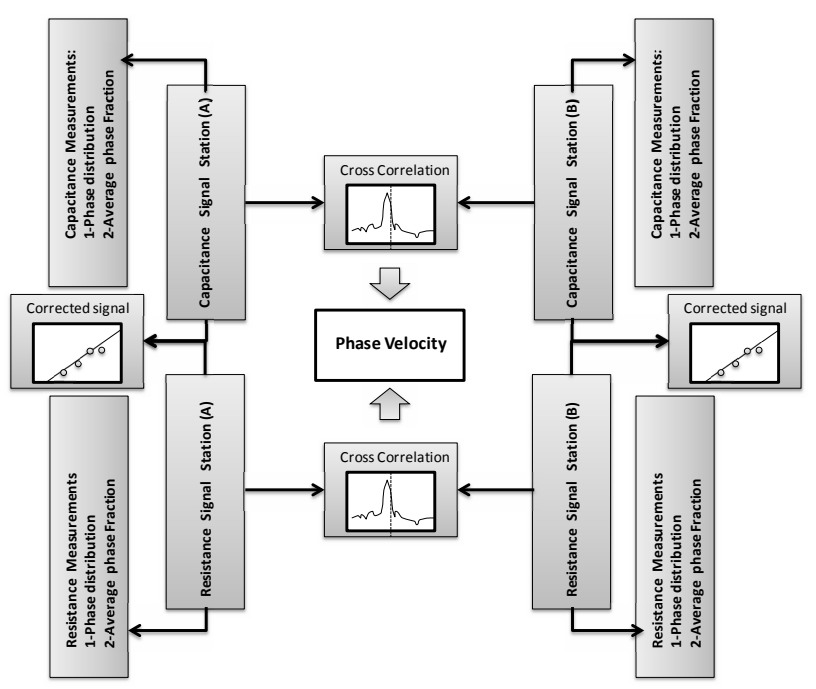

Figure 7: $\quad$ Signal processing chart of the detection device. 


\section{Oil-water two-phase flow measurements using PIV}

In the present study a new device that can be used for the measurement of liquidliquid concentration is design and tested. The device as shown in Figure 8 utilizes the PIV system (1) placed in a casing (2) that houses a transparent section of the pipe (3). The casing is isolated by two opaque plates (4). A photovoltaic (PV) cell (5) is positioned at the other side of the casing facing the light source (1). The PV cell is connected to a data acquisition system (6) for measuring the instantaneous output voltage that depends on the light intensity of the incident beam as shown in Figure 9. In addition, the velocity and vorticity vector can be obtained using the signal processing for the seeding particles in one of the fluid using the PIV system as shown in Figure 9. This light intensity changes with the change of the concentration of the fluid medium. As the oil phase (7) passes through the test section (3), part of the light will be absorbed

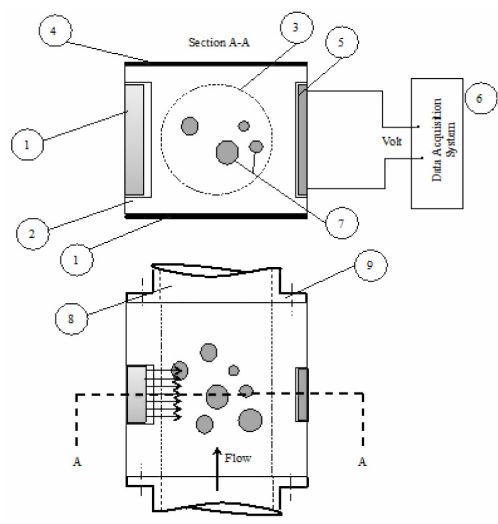

Figure 8: $\quad$ The schematic diagram of the PIV setup.
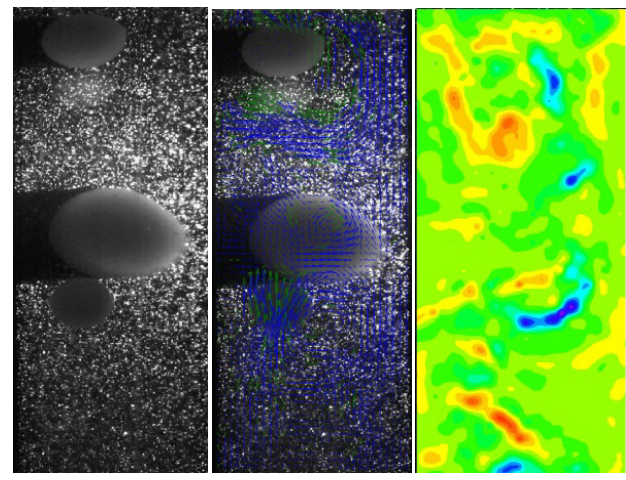

Figure 9: Two-phase structure information using PIV 
resulting in lower light intensity received by the PV cell and consequently decreases the generated voltage. The intensity of the incident light beam will depend on the volume fraction of oil-water two-phase flow. The device is connected to the main piping system (8) through a pipe flanges and bolts (9).

\section{Conclusion}

In comparison with the available tomography techniques previously developed, the present set-up offers continuous operation of both capacitance and resistance probes and, therefore, no high frequency modulator is needed to switch between the capacitance and conductance processes circuits. This will extensively reduce the cost of building such circuit and reduce the error involved due the conditioning process. It should be noted that the cost involved in manufacturing such detection stations is very low compared to available multiphase flow meters.

In comparison with the available optical meters for two-phase flow measurement devices, the proposed PIV system arrangement offers simple measurements of particle concentration in two-phase flow. Available optical sensors cannot be utilized for water cut in oil-water two-phase flow. However, the proposed device is providing a simple relation between the intensity of the light passing through the two phases and the volt generated by the photovoltaic cell.

\section{Acknowledgements}

The authors would like to thank King Abdulaziz City for Science and Technology (KACST) for funding this work under grant No. (MT-5-15). Also, the support provided by the Deanship of Scientific Research (DSR) at King Fahd University of Petroleum and Minerals (KFUPM) is gratefully acknowledged.

\section{References}

[1] Thorn R., Johansen G. A. and Hammer E. A., Recent developments in three-phase flow measurement Meas. Sci. Technol., Vol. 8, No. 7, pp. 691701, 1997.

[2] Wu, Z., McCann, H., Davis, L. E., Hu, J., Fontes, A., and Xie, C. G., (2009), "Microwave-tomographic system for oil- and gas-multiphase-flow imaging”, Meas. Sci. Technol., Volume 20, Issue 10, art. no. 104026.

[3] Warren, P.B., Al-Dusari, K.H., Zabihi, M., and Al-Abduljabbar, J.M., (2005), "Field-Testing A Compact Multiphase Flow Meter - Offshore Saudi Arabia”, Proceedings of the Middle East Oil Show 13, pp. 993-998.

[4] Rajan, V.S.V, Ridley, R.K., Rafa, K.G., (1993), "Multiphase flow measurement techniques - a review", Journal of Energy Resources Technology, Transactions of the ASME, Volume 115 (3), pp. 151-161. 
[5] Zhou, F., Li, Y., Yang, H., Zhang, J., Dong, S., (2006), "Study on multiphase flow online metering models without separation”, Proceedings of SPIE - The International Society for Optical Engineering 6357 I, art. no. $63572 \mathrm{P}$.

[6] Wael H. Ahmed, and B. Ismail, Innovative Techniques for Two-Phase Flow Measurements, Recent Patents in Electrical Engineering Journal, Vol. 1, pp. 1-13, 2008.

[7] Lai, W.T., Bjorkquist, D.C., Abbott, M.P., Naqwi, A.A., Videosystems for PIV recording. Measurement Science and Technology 9, 297-308, 1998.

[8] Tokuhiro, A., Maekawa, M., Iizuka, K., Hishida, K., Maeda, M., Turbulent flow past a bubble and an ellipsoid using shadow-image and PIV techniques. International Journal of Multiphase Flow 24, 1383-1406, 1998.

[9] Brücker, C., Structure and dynamics of the wake of bubbles and its relevance for bubble interaction. Physics of Fluids 11, 1781-1796, 1999.

[10] Oritz-Villafuerte, J., Schmidl, W.D., Hassan, Y.A., Three dimensional PIV study of the surrounding flow and wake of a bubble rising in a stagnant liquid, Experiments in Fluids 29, S202-S210, 2000.

[11] Hassan, Y.A., Oritz-Villafuerte, J., Schmidl, W.D., Three dimensional measurements of single bubble dynamics in a small diameter pipe using stereoscopic particle image velocimetry, International Journal of Multiphase Flow 27, 817-842, 2001.

[12] Merzkirch, W., Gui, L., Hilgers, S., Lindken, R., Wagner, T., PIV in multiphase flow, Proceedings of The Second International Workshop on PIV’97-Fukui, July 8-11, Japan, 1997.

[13] Zhou, Y., Wang, D., Zhang, M., Study on Multiphase Flow and Mixing in Semidry Flue Gas Desulfurization with a Multifluid Alkaline Spray Generator Using Particle Image Velocimetry, Industrial and Engineering Chemistry Research 48, 5808-5815, 2009.

[14] Ahmed, W.H., Capacitance Sensors for Void-Fraction Measurements and Flow-Pattern Identification in Air-Oil Two-Phase Flow, IEEE sensors Journal, Vol. 6 No. 5, pp. 1153-1163, 2006.

[15] Andrade T.L., US patent 2002/000134042, 2007.

[16] Huang SM, Plaskowski AB, Xie CG, Beck MS. Tomographic imaging of two-component flow using capacitance sensors”, J. Phys. E: Sci. Instrum., 22: 173-177, 1989.

[17] Merilo M, Dechene R. L., Cicowlas W. M., Void fraction measurement with a rotating electric field conductance gauge”, J. Heat Transfer Trans. ASME, 99: 330-331, 1977.

[18] Gamio JC, Castro J, Rivera L, Alamilla J, Garcia-Nocetti F, Aguilar L. Visualisation of gas-oil two-phase flows in pressurized pipes using electrical capacitance tomography”, Flow Measurement and Instrumentation, 16: 129-134, 2005.

[19] Jiang T, and Xiong Y., Measurement of the velocity and mass flow rate in gas-solid flow with electrostatic method”, J. Huazhong Univ. of Sci and Tech., 33 (1): 93-95, 2005. 